\title{
Tendencias y perspectivas del estudio de las Relaciones Internacionales: tareas para América Latina
}

Al considerar el desarrollo de las Relaciones Internacionales como disciplina académica, se destaca un hecho fundamental1.

Es solamente en el siglo xx que el estudio de las Relaciones Internacionales comienza a adquirir las características de un estudio académico, y en este proceso, las dos Guerras Mundiales juegan un papel clave.

Al comenzar Ia Segunda Guerra Mundial, en 1939, E. H. Carr publicaba su obra "The twenty years crisis. (1919-1939) An intro-

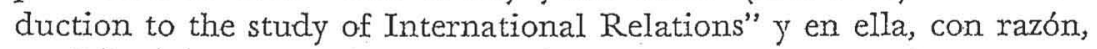
escribía. "La ciencia de la política internacional se encuentra en su infancia"2. Hasta 1914 se consideraba que la guerra era una materia que afectaba a los soldados profesionales y que la política internacional era el dominio exclusivo de los diplomáticos. "No existía el deseo general de sacar la conducción de los asuntos internacionales de las manos de los profesionales, o siquiera de dedicar una atención seria y sistemática a lo que estaban haciendo"s. La' Primera Guerra Mundial disipó por completo esta actitud y en los países de habla inglesa comenzó una campaña de agitación contra los tratados secretos, que eran atacados, con insuficiente evidencia, como una de las causas de la guerra. "Era el primer síntoma de una demanda de popularizar la política internacional y prégonaba el nacimiento de una nueva ciencia"4.

Al intensificarse el desarrollo científico de la disciplina, se generó un intenso debate sobre el alcance, el énfásis y la metodología de las Relaciones Internacionales, dentro del cual se inscribe la querella

ILa expresión "disciplina de las Relaciones Internacionales" se emplea para simplificar. Técnicamente se considera generalmente que las Relaciones Internacionales constituyen más bien un campo de estudio al cual concurren diversas disciplinas de las Ciencias Sociales.

"E. H. Carr, "The 20 years crisis. 1919-1939, An Introduction to the Study of International Relations" (London-New York: Macmillan, 1958) p. I.

ITbid., p. I.

IIbíd., p. 2. 
entre "idealistas" y "realistas". La Segunda Guexra Mundial, al convertir la política internacional en la preocupación diaria del hombre común, dio origen a un movimiento de "popularización" de las Relaciones Internacionales. Al mismo tiempo la relevancia de la variable "poder nacional" enfatizada por los realistas como categoría central de la disciplina, se hizo evidente, no sólo a nivel profesional, sino también a nivel de la opinión pública; dicha relevancia surgía claramente de la emergencia de los Estados Unidos como Ia primera potencia auténticamente mundial y de la unificación planetaria del escenario diplomático. "Desde el Imperio Romano, Occidente no había conocido nada semejante" ". La ascensión de la URss a la categoría de super potencia y la guerra fría no hicieron sino destacar aún más la importancia clave de la lucha por el poder en el escenario internacional.

El movimiento de popularización de las Relaciones Internacio. nales y el profundo cambio de la estructura del sistema internacional, así como las responsabilidades globales de las superpotencias, tuvieron un gran impacto en el desarrollo y en la dinámica interna de la disciplina, generando, especialmente en la universidad norteamericana, un interés creciente en el estudio de las Relaciones Internacionales.

Al mismo tiempo, el estudio del fenómeno internacional hacíase cada vez más complejo por la rapidez del cambio económico, social, político, científico y tecnológico. Al promediar el siglo xx el número de naciones independientes había doblado al existente en 1900; en 1966 alcanzaba a 135 y en 1977 a más de 150 países. A mediados de la década del 60 , el número de organizaciones internacionales llegaba a 1.900, de las cuales sólo 180 eran intergubernamentales. El desarrollo del transporte y de las comunicaciones y el crecimiento de la economía mundial introdujeron cambios substanciales en el carácterde las relaciones internacionales estimulando la emergencia de nuevos fenómenos como el de la integración económica regional. Los cambios en la economía mundial crearon, a través de las corporaciones transnacionales, el fenómeno de la internacionalización de la produc. ción, de naturaleza mucho más compleja que el de la internalización del comercio. EI desarrollo científico y tecnológico y la creación de nuevas armas despertaron un interés creciente de académicos de las ciencias naturales en el estudio de las Relaciones Internacionales. Las p. 13 .

"Raymond Aron, "Paix et Guerre entre les nations" (Paris: Calman-Lévy; 1962) 
armas nucleares alteraron el rol tradicional que la violencia había jugado en la resolución de los conflictos internacionales. La conciencia de los límites de la tierra ante las demandas de un crecimiento indefinido y la crisis energética hicieron surgir el nuevo concepto de politica de la escasez. La crisis del sistema económico creado en Bretton Woods, la crisis ecológica, la crisis de alimentos, etc. transformaron tan dramáticamente el carácter del sistema internacional que "aún el vocabulario de las Relaciones Internacionales rápidamente se convirtió en obsoleto"6.

Dichos cambios y la complejidad del fenómeno internacional hicieron que "hombres de virtualmente todas las disciplinas empezarán a contribuir al estudio de las Relaciones Internacionales"

El cambio científico no solamente afectó el estudio de las Relaciones Internacionales a través del impacto del cambio tecnológico sobre el tipo de datos utilizados por la disciplina, sino que también incluyó en las técnicas analíticas. En este proceso tuvo gran relevancia el progreso experimentado en las Ciencias Sociales en general. Surgió una nueva generación de especialistas en Relaciones Internacionales que se beneficiaron con dicho progreso, iniciaron una etapa de colaboración interdisciplinaria, y empezaron a realizar significativos avances hacia el desarrollo de una ciencia de las Relaciones Internacionales. Los conceptos y técnicas empleados en analizar tópicos tales como la adopción de decisiones, teoría de los juegos, análisis de sistemas, simulación, geografía, actitudes, estrategia militar, investigaciones sobre la paz (peace research) etc. fueron aplicados al campo de las Relaciones Internacionales.

Al mismo tiempo, la variable "poder nacional" implantada por los "realistas" como categoría central de análisis empezó a ser cuestionada desde el punto de vista analítico pues se estimó que tal categoría era demasiado comprehensiva y no podía explicar una variedad de fenómenos como, por ejemplo, la influencia ejercida en determinadas circunstancias por naciones menores sobre países que aparecían con mayor poder dentro de los análisis "convencionales". Una investigación publicada en 19.63 encontró que el concepto de poder era usado en 17 acepciones diferentes en textos de estudio y que numerosos autores daban al concepto usos muy diversos ${ }^{8}$.

International Encyclopedia of the Social Sciences, volumen 8, p. 62.

Tbid., p. 62.

${ }^{8}$ Denis Sullivan, "Toward an inventory of mayor propositions contained in contemporary textbooks in. International Relations". Ph. dissertation, Northwestern University, 1963. 
Al considerar esta evolución en perspectiva, es posible distinguir en el desarrollo de la disciplina en los Estados Unidos diversas corrientes que, aunque se superponen y se entrecruzan en el tiempo, se manifiestan en tendencias que pueden considerarse predominantes en ciertos períodos.

La orientación normativa propia de los estudios jurídico-internacionales cede el paso en la década del 40 a la orientación de la escuela realista en que prevalece el estudio empírico de los fenómenos internacionales, estudio centrado en torno a las nociones claves de interés nacional y poder nacional. En las décadas del 50 y del 60 predomina crecientemente la orientación conductualista influenciada grandemente por el desarrollo similar de otras Ciencias Sociales y por la aplicación de los métodos cuantitativos ${ }^{9}$.

La escuela conductualista ha desplazado el centro de la preocupación desde la prescripción, la investigación ética y la acción hacia "la descripción, la explicación y la verificación", (como expresó David Easton). En la década del 60, el conductualismo ha sido la tendencia predominante.

En la década actual las Relaciones Internacionales parecen estar entrando a una cuarta etapa, denominada postconductualista, en la cual emergen diversas tendencias que conviene precisar para definir el ámbito de sus preocupaciones en el país en que ella ha alcanzado su mayor desarrollo ${ }^{10}$.

Se han producido en el campo de la disciplina, nuevas controversias que han reemplazado al antiguo debate entre "idealistas" y "realistas". Una de ellas es de carácter puramente académico y versa, no sobre temas sustantivos -la materia de estudio- sino sobre los métodos de análisis. Es la controversia entre "tradicionalistas" y "científicos" (conductualistas). Una segunda se refiere a una nueva revolución experimentada en la Ciencia Política, disciplina intimamente ligada a la de las Relaciones Internacionales. Una tercera incide en el impacto que el emergente campo de los estudios sobre el futuro han tenido en la disciplina (future studies), y finalmente una cuarta de carácter netamente político, se refiere a la búsqueda de un foco o núcleo orientador de la política exterior de los Estados

'James E. Daugherty y Robert L. Pfaltzgraff, "Contending Theories of International Relations" (New York: J. B. Lippincot Co. 1971) p: 379.

${ }^{10}$ Hedley Bull, "International Theory: The case for a classical approach" en Klatis Knoff and James $N$. Rosenau "Contending Approches to International Politics" (Princeton, New Jersey, Princeton University Press, 1969) pp. 20-39. 
Unidos. Es la controversia entre "realistas del poder" y "humanistas planetarios".

Ninguna de estas controversias podría entenderse sin referirse al contexto en que ellas se desarrollan y que está constituido por la crisis generalizada del sistema internacional, y la crisis interna que ha sufrido Estados Unidos bajo el triple impacto de Vietnam, Watergate y del affaire de la ciA.

El debate entre "tradicionalistas" y "científicos" constituye un cuestionamiento de los primeros a los métodos de la escuela conductualista, expresados en siete proposiciones críticas enunciadas por Hedley Bull en su polémico artículo "International theory. The case for a classical approach" publicado por World Politics en 1966. La acusación principal de Ios "tradicionalistas" es que, al circunscribir sus estudios a lo que puede ser probado lógica o matemáticamente o verificado de acuerdo con procedimientos estrictos, los que practican el "método científico" se están negando a sí mismos los únicos instrumentos actualmente disponibles para desentrañar la materia en estudio. Al abstenerse del empleo del enfoque intuitivo o de la "sabiduría literaria" (filosofía, historia iv derecho) los "científicos" se condenan a una especie de puritanismo intelectual que los mantiene alejados de la substancia de la política internacional. Cuando ocasionalmente arrojan Iuz sobre dicha substancia, es porque realmente han empleado el método clásico o tradicional; en cambio, el empleo del "método científico" sólo los lleva a tratar tópicos periféricos a la disciplina, los cuales en algunos casos se ven distorsionados o empobrecidos por el fetiche de la medición cuantitativa. De esta manera los "científicos" han menoscabado la teoría al concebirla como la construcción y manipulación de los llamados "modelos". El rigor y la precisión que requiere la teoría de la política internacional puede ser alcanzado por los métodos clásicos. Así lo prueban las obras de académicos como E. Carr, Hans Morgenthau, Georg Schwarzenberger, Raymond Aron, etc. En cambio, los que practican el "método cientifico", al separarse de las corrientes de la historia y la filosofía, se privan de los medios que les permitirian ejercer su propia autocrítica $y$, en consecuencia, tienen de las Relaciones Internacionales, una visión que es aventurada y estrecha ${ }^{11}$.

Intimamente vinculado con este debate se encuentra lo que David Easton denomina la Nueva Revolución de la Ciencia Política, que

IDavid Easton, "The New Revolution in Political Science" en The American Political.Science Review, Vol, Lxm, December.1969, No 4. 
marcaría el comienzo de una nuevà corriente de las Ciencias Sociales denominada también postconductualista ${ }^{12}$.

La emergencia de esta nueva corriente se explica sobre todo por la crisis generalizada del sistema internacional y de la crisis norteamericana.

Para Easton, debido a esta crisis, la humanidad está trabajando bajo la presión del tiempo, El tiempo ya no está en favor del hombre y este hecho constituye algo nuevo y pavoroso en los asuntos mundiales. Las tensiones provocadas por las crisis generatizadas se mueven en una dirección cierta: conflictos sociales crecientes y crecientes temores y ansiedades sobre el futuro, no sólo de una generación o de una nación, sino de la raza humana misma.

Frente a una situación semejante, el movimiento postconductualista de la Ciencia Política levanta dos banderas: relevancia y acción. Reclama investigaciones más relevantes y una orientación hacia el mundo que induzca a los politólogos aún en su capacidad profesional, a prescribir y actuar para mejorar la vida política en su sentido más amplio, de acuerdo con criterios humanos. El credo del nuevo movimiento podría sintetizarse en los siguientes rasgos que en su conjunto representan una especie de tipo ideal de desafíos a la escuela conductualista: 1) La substancia debe preceder a la técnica. Si una debe ser sacrificada en beneficio de la otra -y ello no será siempre necesarioes más importante ser relevante y significativo con respecto a los urgentes problemas sociales contemporáneos, que ser sofisticado en cuanto a los instrumentos de investigación. Frente al aforismo de la ciencia que sostiene que es mejor estar equivocado que ser vago, el postconductualismo establecería un nuevo dictum: es mejor ser vago que ser preciso y no relevante; 2) La escuela conductualista oculta una ideología de conservantismo empírico, ya que circunscribirse a la descripción y análisis de los hechos es dificultar la comprensión de estos hechos en un contexto más amplio, favoreciendo así el man. tenimiento de las mismas condiciones factuales que se exploran. De esta manera, aunque involuntariamente, se favorece una ideología de conservantismo social, temperada únicamente por modestos cambios incrementales; 3) el corazón de la investigación conductualista es la abstracción y el análisis y ello sirve para ocultar las crudas realidades de la política. La tarea del postconductualismo es romper las barreras del silencio que el lenguaje conductualista ha necesariamente creado y ayudar a la Ciencia Política a enfrentar las necesi-

InIbid., pp. 1051-1061. 
dades reales de la humanidad en un tiempo de crisis; 4) la investigación sobre Ios valores y el desarrollo constructivo de ellos, forman parte del estudio de la política. La ciencia no puede ser y nunca ha sido neutral desde el punto de vista valorativo a pesar de las protestas en contrario. En consecuencia, para entender los límites de nuestro conocimiento, necesitamos estar conscientes de las premisas de valor sobre las que se basa y de las alternativas para. las cuales puede ser usado; 5) los miembros de una disciplina académica tienen la responsabilidad de todos los intelectuales. EI rol histórico de los intelectuales ha sido y debe ser el de proteger los valores humanos de la civilización. Esta es su peculiar tarea y obligación. Sin ello se convierten en simples técnicos, en mecánicos para remendar chapuceramente la sociedad; 6) el conocimiento conlleva la necesidad de actuar, y actuar es comprometerse en la configuración de la sociedad. El intelectual, como científico, tiene la obligación de emplear su conocimiento. La ciencia contemplativa era un producto del siglo xrx, cuando un concenso moral más amplio era compartido. La ciencia activa refleja necesariamente el conflicto en la sociedad contemporánea sobre los ideales y ello debe permear e iluminar toda la tarea investigadora; 7) si el intelectual tiene la obligación de implementar su conocimiento, las organizaciones profesionales de intelectuales y las mismas universidades no pueden permanecer al margen de las luchas cotidianas. La politización de las profesiones es tan inescapable como deseable ${ }^{13}$.

La orientación hacia la relevancia y hacia la acción establecen un claro vínculo entre "la nueva revolución en la Ciencia Política" y los estudios del futuro, nuevo campo que ha emergido con inusitado vigor en los últimos años. En efecto, si hay una tendencia que caracteriza a los estudios del futuro es el afán de escudriñar el porvenir en términos de hacer frente a las crisis multifacéticas que aquejan al sistema internacional y -a través de planteamientos utópicos-normativos- poder aportar solución a los problemas planetarios que de ellas derivan. En consecuencia, estos estudios están decididamente orientados a la relevancia y a la acción tanto en el plano intelectual como polf́tico para modificar más o menos radicalmente una realidad que se juzga insatisfactoria.

13Ibíd., p. 1058. Sobre los estudios utópico-normativos, ver la serie de 6 libros publicados por The Free Press (Nueva York), titulada "Preferred Worlds for the 1990's"; ver, por ejemplo, Gustavo Lagos and Horacio Godoy "Revolution of Being. A Latin American view of the future" (New York, The Free Press, 1977). 
Estos planteamientos utópico-normativos descansan en una especu. lación creativa que integra modelos de pensar normativos, descriptivos, teóricos y conductualistas en una metodología que se encuentra aún en plena gestación. Este enfoque multimetodológico acerca así a los estudios del futuro con la tendencia de la nueva revolución en la Ciencia Política, ya que esta también propicia la "especulación creativa" sobre las alternativas políticas como una manera de señalar no sólo las deficiencias de los actuales sistemas políticos, sino también de explorar posibilidades de cambio que tan urgentemente se requieren ${ }^{14}$.

Por último, cabe referirse a las dos escuelas antagónicas de pensamiento que representan hoy día las principales líneas de división entre el público interesado e informado dentro de Estados Unidos con respecto a la búsqueda de un foco de orientación para la política exterior de la gran potencia. Como escribe Zbigniev Brzezinski, actual asesor de Seguridad Nacional del Presidente Carter, en un artículo publicado en 1973 en la revista "Foreign Affairs", la visión de estas dos escuelas en competencia puede ser etiquetada con los rótulos de "realismo del poder" y "humanismo planetario"15.

Los realistas del poder, generalmente más conservadores en sus valores, tienden a ocuparse de las inquietudes más tradicionales de las cuestiones internacionales, particularmente de problemas tales como estrategia, relación de fuerzas, equilibrio de poder, diplomacia y política monetaria; ellos atribuyen un valor muy alto a la estabilidad tanto como concepto que como norma. A menudo pueden ellos diferir en lo relativo a prescripciones y prioridades, pero mantienen un punto de vista básico común en cuanto visualizan el mundo como todavía dominado por la política internacional.

En contraste, los humanistas planetarios tienden a considerar al mundo como una "unidad" acosada por ciertos problemas comunes. Al intentar traducir en políticas sus predisposiciones básicas, ellos tienden a concentrarse en materias tales como ecología, nutrición, desarrollo, justicia social e igualdad o límites del crecimiento. Existen pues reminiscencias del tomo de este debate con el de la vieja querella entre "realistas" e "idealistas", pero las diferencias son im. portantes; los nuevos realistas del poder aceptan cada vez más la noción de interdependencia política, y su interés por la estabilidad hasta cierto punto supera a la anterior preocupación de los idealistas

"Zbigniew Brzezinski, "U.S. foreign Policy: a search for focus", en Foreign Affairs, July, 1973.

${ }^{15}$ Ibid. 
por la paz. Los humanistas planetarios, a diferencia de sus predecesores, los idealistas, se interesan mucho más por el cambio social que por la paz en un mundo que ven acosado por crisis socioeconómicas, dinámicamente crecientes, y concentran sus remedios en reformas sociopolíticas. Muchos de ellos restan méritos a la estabilidad y, en algunos casos, llegan incluso a aceptar la conveniencial del cambio violento ${ }^{16}$.

Los realistas del poder defienden una buena causa cuando sostienen que el restarle importancia al poderío nacional, a la diplomacia y a sus herramientas más tradicionales del comportamiento internacional podría poner en peligro la posibilidad de la paz e impulsar la inestabilidad internacional. Están también en lo correcto cuando alegan que una concentración exclusiva en las grandes cuestiones planetarias, si bien mucho más atractiva moralmente hablando, ignora las realidades de un mundo de naciones-estados, un mundo de realidades nacionalistas, de ejércitos nacionales y de hostilidades ideológicas. Aunque no sea aislacionista en espíritu, el humanismo planetario puede ser acusado de ser esencialmente escapista.

Sin embargo, aquellos que insisten en el humanismo planetario ésgrimen un poderoso argumento cuando advierten que ignorar la amenaza a largo plazo (de los problemas planetarios) es invitar, para dentro de una o dos décadas, a una situación de anarquía mundial, de fragmentación de las instituciones políticas y sociales, de colapso de la estabilidad a la que los realistas del poder atribuyen tan alto valor ${ }^{17}$.

A pesar de estas diferencias básicas, los realistas del poder y los humanistas planetarios mantienen todavía un acuerdo fundamental en el sentido de que la interdependencia mundial - sin tomar en consideración qué prioridad se asigna a la seguridad política o al bienestar social- es la inescapable realidad de nuestro tiempo ${ }^{18}$.

$\therefore$ Las observaciones anteriores revelan que en las cuatro grandes controversias - algunos de cuyos aspectos hemos esbozado- el impacto de la crisis multifacética del sistema internacional se hace sentir en términos de que tanto para los humanistas planetarios, y para los estudiosos del futuro, como para los que propician la nueva revolución de la Ciencia Política, Ia relevancia de la temática estu-

IeIbid.

ITbid.

${ }^{19}$ José A. Silva Michelena, "Política y Bloques de Poder. Crisis en el Sistema Mundial” (México, Siglo xxu Editores, 1976) p. 16. 
diada se juzga en función de su relación directa con los problemas planteados por dichas crisis, y en estas tres corrientes está explícitamente establecido el compromiso con la acción en pro de un cambio social que privilegie los valores humanos.

Al mismo tiempo, el análisis anterior demuestra el estado generalizado de insatisfacción con el desarrollo de la disciplina, que se. traduce en la multiplicidad de enfoques para su estudio, en la multiplicidad de teorías existentes y en la multiplicidad de métodos propiciados. Ante este pluralismo de orientaciones, la tendencia general parece ser la de propiciar la complementariedad de los enfoques como. una manera de enriquecer la disciplina de las Relaciones Internacionales.

Cualquiera que sea el grado controversia e insatisfacción que envuelve el desarrollo de la disciplina de las Relaciones Internacionales en Estados Unidos, es preciso constatar que el número y la calidad de los esfuerzos en el plano de la docencia y de la investigación ha alcanzado un alto nivel académico que sería difícil de igualar en otros países industrializados que han jugado o juegan un papel importante en la política internacional como Inglaterra, Francia y la República Federal Alemana. En la URss los estudios sobre Relaciones Internacionales siguen las líneas tradicionales de la interpretación marxista-leninista sobre el imperialismo y, al parecer no "han abordado apropiadamente la cuestión de la creciente heterogeneidad del mundo, como consecuencia de la expansión del campo socialista. Esta omisión. era quizás aceptable hasta la Segunda Guerra Mundial, ya que el. aislamiento de la Unión Soviética y su relativamente bajo peso específico en la política mundial afectaba muy poco los análisis que se hacían. Sin embargo, a partir de la Segunda Guerra Mundial el campo socialista se expande significativamente, de modo que incide tanto sobre la propia política de los países imperialistas, como sobre los países subdesarrollados. Como consecuencia de ello, los análisis de política internacional que se basan exclusivamente en la teoría del imperialismo son, por decir lo menos, incompletos. Más aún, puede decirse sin temor a equivocaciones que dentro del marxismo aún no ha surgido una teoría de las relaciones entre los campos socialista y capitalista, para no hablar de las relaciones intersocialistas"19.

${ }^{19}$ Alberto Sepúlveda "Problemas en el desarrollo de los estudios sobre Relaciones Internacionales en Chile" en Revista Estudios Sociales, No 8, Junio, 1976, Santiago, Chile, p. 30. 
Gon respecto a América Latina, el primer hecho que se destaca es que la región carece de una dotación importante y adecuada de instituciones de docencia e investigación que le permita contar con los especialistas y los estudios necesarios para la formulación y conducción de su política internacional ${ }^{20}$. La situación puede variar de país a país y el fenómeno presentarse con mayor o menor intensidad, pero es indudable que ésta es la situación general.

EI desarrollo de las Relaciones Internacionales como disciplina académica es incipiente $y$, salvo el caso de especialistas aislados cuya existencia sólo confirma la regla general, hasta fecha muy reciente la disciplina estuvo circunscrita a estudios de carácter histórico y legal, particularmente de este último tipo. Basta señalar, para ello, que el Instituto de Estudios Internacionales de la Universidad de Chile sólo se crea en 1966 y que la primera Cátedra de "Política y Relaciones Internacionales" en la misma Universidad, sólo data de 1970.

Las razones explicativas de esta situación se encuentran en dos tipos de causas, una de carácter académico vinculada al desarrollo de las Ciencias Sociales y particularmente, de la Ciencia Política en la región, y otra de carácter histórico internacional. Con respecto a la primera, cabe señalar que el desarrollo de la Ciencia Política en la región ha sido escaso y precario. Como el estudio de las Relaciones Internacionales ha estado estrechamente relacionado con la Ciencia Política, el subdesarrollo de ésta ha privado a los estudios internacionales de nuevos enfoques metodológicos, conceptuales y substantivos.

Con respecto a la segunda causa, ya se ha señalado que el desarrollo de la disciplina de las Relaciones Internacionales entró en una fase de gran avance en los Estados Unidos debido a los requerimientos impuestos por la formulación y conducción de su política exterior $y$ es un hecho que, en general, la disciplina ha cobrado auge en las naciones que han jugado un papel activo en la política y economía mundiales ${ }^{21}$.

En América Latina, por más de un siglo, los únicos dedicados al

${ }^{20} \mathrm{El}$ profesor Claudịo Véliz ha señalado que los primeros centros de eștudios internacionales fueron creados en Gran Bretaña, Francia y Estados Unidos inmediatamente después de concluidas las negociaciones de paz de Versalles. El Royal Institute of International Affairs de Londres tenía como indisimulado propósito contribuir a la.paz mundial.

Mlandio Véliz: "E1 Instituto de Estudios Internacionales de la Universidad de Chile" en Revista de Estudios Internacionales, Ni 1, 1967, p. 4. 
cultivo de la disciplina, aparte de historiadores y juristas como se ha indicado, fueron los encargados de conducir la diplomacia, los cuales, al decir de un autor, estaban empeñados en una compleja actividad circunscrita a asuntos intralatinoamericanos, en una especie de microcosmos de la política mundial, con' guerras internacionales, pactos defensivos y ofensivos, tratados secretos, arbitrajes, $y$ toda unaserie de instrumentos imitados de la vieja diplomacia europea.

Guando Estados Unidos inició su acción imperialista a fines del siglo $\mathrm{xIx}$ y en las primeras décadas de este siglo, la acción internacional de América Latina se concentró en las relaciones interamericanas para defenderse del Coloso del norte, al mismo tiempo que continuaba desarrollándose un sistema de balanza o equilibrio de fuerzas militares en escala local.

Durante la Primera Guerra Mundial, Ios principales países de América Latina observaron una estricta neutralidad, lo que los manmantuvo relativamente aislados del primer gran conflicto.

El ingreso de algunos países de la región a la Sociedad de las Naciones y el debate generado en torno a ella, el ingreso masivo a la Organización Internacional del Trabajo y la crisis de 1929, ensancharon el panorama internacional de las principales naciones del área, que empezaron a mirar y a ocuparse en forma incipiente de los asuntos de la política mundial. Es sintomático, por ejemplo, que el primer centro para el estudio de los asuntos internacionales de que se tenga memoria en América Latina, de efímerã existencia, fuera fundado por un grupo de chilenos a mediados de la década de $1930^{22}$.

La Segunda Guerra Mundial congeló esta situación, al dividirse el planeta en dos bloques al parecer irreconciliables. Sobre las ruinas del antiguo, se generó un nuevo sistema internacional altamente estratificado, con las super potencias en su cúpula, que ejercen su hegemonía sobre las zonas de influencia delineadas en YaIta. El deterioro del status internacional de las potencias tradicionales - Inglaterra, Francia, Alemania y Japón- generalizó el fenómeno de la. dependencia internacional.

En este período se producen diversos hechos que configuran un nuevo desafío externo para América Latina, pero de naturaleza muy distinta a la del primer desafío externo - la lucha por la indepen-

${ }^{23}$ Claudio Véliz, ibíd. 
dencia y la construcción de las nuevas nacionalidades y al segundo desafío- Ia lucha contra el imperialismo del "gran garrote". El nuevo desafío está configurado por una constelación de nuevos hechos y situaciones: 1) la toma de conciencia de la división del mundo en países desarrollados y subdesarrollados, nociones que son difundidas por las Naciones Unidas, a la cual han ingresado masivamente todos los países latinoamericanos. El fenómeno había empezado a surgir doscientos años atrás con la revolución industrial inglesa y su difusión posterior a Europa Occidental, los Estados Unidos y el Japón; pero es sólo en la década de 1960 cuando surge la conciencia de este hecho fundamental; 2) América Latina es clasificada en el mundo del subdesarrollo, el cual, a partir de la década del 60, es denominado Tercer Mundo; 3) América Latina es la única región subdesarrollada de Occidente; 4) en la Guerra Fría, América Latina se alínea con Estados Unidos, entra en un pacto de alianza militar expresado en el TIAR (Tratado Interamericano de Asistencia Recíproca) y las relaciones interamericanas se institucionalizan en la OEA. La política del Buen Vecino de Franklin D. Roosevelt ha terminado y América Latina pasa a ser un aliado de segunda categoría cuyo apoyo Estados Unidos da por descontado, hasta que surge la revolución cubana y su contrapartida, la Alianza para el Progreso; 5) el mundo subdesarrollado toma como modelo implícito o explícito el modelo de desarrollo de las superpotencias, en su versión capitalista norteamericana o en su versión socialista soviética.

Como se ha dicho, con todos estos hechos, ha surgido para América Latina un nuevo desafío externo: lograr el desarrollo y superar su dependencia. Pero a diferencia de los desafíos anteriores, este desafío externo es de un carácter difuso, no tiene ni la concreción de las guerras de la independencia, ni la concreción tampoco del imperialismo del gran garrote; es captado por las élites y no por las grandes mayorías.

Las formas de dominación se han hecho más sutiles y aún se disfrazan de "ayuda internacional" económica, técnica y militar. A las formas de dominación comercial basadas en la división internacional del trabajo forjada en el siglo xrx, se agregan, ahora, nuevas formas de dominación representadas por la internacionalización o transnacionalización de la producción a través de las corporaciones transnacionales.

Este desafío externo es captado por una élite intelectual latinoamericana, proceso que se inicia con la toma de conciencia operada 
a través de la GEPAL y que se amplía luego a través de los trabajos de la UNCTAD, del BID, de la GECLA, etc. Surgen así nuevas generaciones de latinoamericanos -políticos, intelectuales, funcionarios técnicos nacionales e internacionales- que van definiendo y precisando los alcances y proyecciones del desafío.

Empieza a generarse así un proceso de autoconciencia latinoamericano, que se expresa a través del movimiento de integración económica en sus distintos esquemas en cuanto a la política exterior intralatinoamericana, $y$ en la cecLA en cuanto a la coordinación de las politicas exteriores. En la década de 1960, se crean la olEP y el GLPEP con lo cual América Latina inicia también su apertura a otros países del Tercer Mundo para la defensa de sus intereses comunes en el campo de las materias primas. El Consenso Latinoamericano de Viña del Mar, adoptado en el marco de GECLA, marca un hito en el desarrollo de esta autoconciencia a fines de la década del 60. La organización del grupo de los 77 y la emergencia del bloque de los no alineados significan la irrupción a nivel del Tercer Mundo de una clara actitud combativa en el plano internacional de América Latina, de Asia y de Africa.

La transformación del sistema internacional bipolar en un sistema multipolar y el proceso de détente marcan el fin del marco rígido de la guerra fría, cambios que dan al panorama internacional una gran fluidez, creando un margen de movilidad para los países medianos y pequeños que les permite cierta autonomía en sus decisiones y actitudes internacionales.

Todos estos fenómenos llevan a un resurgimiento de los nacio. nalismos, a un cambio en la mentalidad Iatinoamericana, que sale de su actitud pasiva para emerger como una fuerza activa en la política mundial, ya sea por sí sola o en alianzas múltiples en el seno del Tercer Mundo, en el grupo de los no alineados, en las asociaciones de productores de materias primas y en organismos internacionales gubernamentales y no gubernamentales, cuya proliferación cons. tituye una verdadera explosión de contactos internacionales de todo tipo.

Se genera así un movimiento de "popularización" del interés por las Relaciones Internacionales, que en las décadas del $60 \mathrm{y}$ del $70 . \mathrm{se}$ traduce en demandas por un conocimiento objetivo de las complejas interacciones políticas, económicas, culturales y científico-tecnológicas entre las naciones de nuestra región y los países del resto del mundó.

Es en este contexto que hay que situar las perspectivas del desa- 
rrollo de la disciplina de las Relaciones Internacionales en América Latina. Si bien es cierto que la región carece, como se anotó más arriba, de una dotación de instituciones de docencia e investigación en este campo, es indudable que ya ha emergido claramente la convicción de esta insuficiencia, al menos al nivel académico.

El momento, parece, por tanto, altamente propicio para emprender un vasto esfuerzo a nivel regional en pro de un desarrollo científico de la disciplina.

Tal esfuerzo debería revestir, a nuestro juicio, Ias siguientes características generales:

1) El desarrollo moderno de la disciplina exige la integración y aporte de diversas ramas del conocimiento y en especial de las ciencias sóciales como la Sociología, la Ciencia Política, la Psicología Social, là Economía, la Ecología, en enfoques interdisciplinarios, que superen los simples enfoques legales, periodfsticos, históricos y diplomáticos del pasado,

2) EI estudio de las Relaciones Internacionales en América Latina debería evitar lás muchas veces inútiles querellas que se han producido en Estados Unidos en el desarrollo de la disciplina y que se han señalado anteriormente, pues resulta evidente a la luz de esa experiencia que corresponde emplear una multiplicidad de enfoques teóricos y metodológicos, ya que unos y otros son complementarios. Es igualmente claro que los proyectos de investigación que se seleccionen deben ser relevantes desde el punto de vista de los intereses de América Latina y deben estar orientados a maximizar la presencia de la región en el sistema internacional con miras a la construcción de un nuevo orden mundial;

3) Dentro de la tarea anterior, se requere un esfuerzo creativo de tipo teórico que permita situar a América Latina en su conjunto y a lös diversos países que la integran en su inserción en el sistema internacional;

4) Es preciso crear nuevos ámbitos de estudio, aunando y coordinando el esfuerzo de centros y especialistas de los distintos países de la región;

5) Es preciso integrar el esfuerzo de investigación con el de forma- 
ción de especialistas a fin de obtener una fecundación recíproca entre ellos y;

6) En el diseño de los programas de investigación hay que partir del hecho de que la disciplina de las Relaciones Internacionales ha sido desarrollada casi enteramente en Estados Unidos y otros países centrales, y que el cuerpo de conocimiento teórico existente refleja los intereses y las concepciones de los países dominantes en el sistema internacional. Por tanto, existe la necesidad de proceder, en la tarea investigadora, a una revisión crítica del conocimiento disponible tanto desde el punto de vista de su base teórica como del examen empírico de la realidad.

Tales son las tareas que enfrenta el desarrollo de la disciplina de las Relaciones Internacionales en América Latina, y a la comunidad académica de la región le corresponde hacer frente a este desafío intelectual. 